\title{
GPS \& GIS In Road Accident Mapping And Emergency Response Management
}

\author{
Evangeline Muthoni Njeru ${ }^{1}$, Andrew Imwati ${ }^{1}$ \\ ${ }^{1}$ Jomo Kenyatta University of Agriculture and technology, Department of Geomatic Engineering \\ and Geospatial Information Systems
}

\begin{abstract}
Road accidents is a major cause of mortality worldwide with urgent action required to mitigate against the negative impacts. A comprehensive accident recording and analysis system can provide a GISbased solution for control and management of accident events as a real-time monitoring system. This paper presents a GIS approach to road accident management based on spatial autocorrelation. It goes further to analyzing the spatial viability of emergency services offered to the accident victims. The study starts with the identification of the accident prone zones along Waiyaki Way in Nairobi, Kenya using GPS and GIS. For this purpose, the road accident data for the years 2013, 2014 and 2015 pertaining to Waiyaki Way was obtained from the traffic police department and used for analysis. Accident particulars like date, location, number of victims (fatal, serious and slight injuries), classes of victims (drivers, motor cyclists, pedal cyclists, passengers, pedestrians) were included in the GIS database. The "Density" function available in the spatial analyst extension of the Arc GIS software was applied to identify the accident prone areas during the years 2013, 2014 and 2015. Assessment of spatial clustering of accidents and hotspots spatial densities was carried out following Moran's I method of spatial autocorrelation, and point Kernel density functions. An effort was made to develop a comprehensive accident information management system that can provide a GIS-based solution for control and management of accident events. This system will inform clients about accident locations, accident and service diagnosis, reducing the number of accidents based on the accident reports thus increasing the level of road safety and fast delivery of emergency services.
\end{abstract}

Keywords; Accidents, GIS, AIMS

\section{Introduction}

According to the WHO, over 1.2 million people die each year due to road accidents, with about 20 and 50 million suffering from non-fatal injuries. More damning and intriguing is that despite the increasing sensitization on roads safety worldwide, the epidemic of road accidents is still on the rise. In the last five years alone, most countries adopted the WHO report on the road safety guidelines(WHO 2009). But there are still increasing trends of road accidents. Urgent action is needed to achieve the ambitious target for road safety reflected in the newly adopted 2030 Agenda for Sustainable Development, of reducing by half the global number of deaths and injuries from road traffic crashes by 2020(Lagarde 2007).

African countries especially those in the East African region have some of the worst rates and incidences of road accidents(Odero et al. 1997). Kenya alone has approximately 29.1 deaths per 100,000 people(Muchene 2014). This is among the worst 20 in the world with traffic accidents in the country leading to more deaths that the endemic malaria. Kenyan government has spent millions of money in order to reduce the number of accident occurrence through several modes of campaign. Unfortunately, the number keeps increasing. An effective accident management tool and model is therefore required to help manage accidents and mitigate against its negative influences (Manyara 2015).

This paper presents a GIS approach that can be used for the management of road accidents in Nairobi, Kenya such as the one presented in(Rudtanasudjatum 2008). GIS has wide applications including health (Alegana et al. 2014), climate (Ouma et al. 2016), transportation(Nagne and Gawali 2013), forestry among others. We intend to harness some of these capabilities in the area of accident management systems. The spatial viability of the emergency services to common accident hotspots is also assessed. Accident hotspots along one of Kenya's busy highways i.e. Waiyaki way are defined from existing traffic databases. In our analysis, road accidents data for the years 2013, 2014 and 2015 are obtained from the traffic police department. Accident particulars like date, location, number of victims (fatal, serious and slight injuries), classes of victims (drivers, motor cyclists, pedal cyclists, passengers, pedestrians) were included in the GIS database. The "Density" function available in the spatial analyst extension of the Arc GIS software was applied to identify the accident prone areas during the years 2013, 2014 and 2015. Both simple and Kernel densities was applied in identifying the accident patterns. 


\section{Data and sources}

\section{Methods}

Accident data was sourced from the Kenya Police Traffic Crash Reports (TCR) for 2013, 2014 and 2015. The reports are compiled by the Kenya police on the daily accident occurrences including information on where the accident occurred, the timing, causes, fatalities, outcomes and whether victims were taken to hospital. Health facility data was compiled by using two sources that exist for the country. One is health facilities data from Kenya open data platform which was downloaded on December 2015 at (https://opendata.go.ke). The second health facility database was obtained from the list compiled by (Noor et al. 2009). The two sources were cross checked for any inconsistencies especially for the Nairobi area, and one final list of health facilities was obtained. Information on the level of care and location of the health facilities was also attached to our final file. Roads data was obtained from the Kenya roads board that maintains the most up to date and current roads inventory in the country. The data was compiled in 2009 where GPS receivers were used to collect roads data including the coverage, classes of the roads and conditions. This was deemed more accurate compared to the openly provided road networks from sources such as open street maps. County boundaries were obtained from Independent Electoral and Boundaries Commission (IEBC) which is mandated to maintain the boundary data for the whole country.

\section{Data Cleaning}

The road accidents data had inconsistencies in both the attribute and location information. Editing was therefore done by cross checking the attributes in Microsoft Excel to ensure that all the attributes used were correct. In addition the latitude and longitude were assigned to each accident location through the process of geocoding using various online mapping tools such as google maps and open street maps. All vector data (i.e. line, polygon, point features) contained separate attribute tables. The final accident data contained information on; Date, Location and time of occurrence, type of accident, type of injury, fatalities, the victims or the actors, the health facility that responded to the injured parties. Descriptive statistics of data on accidents were produced using graphs and pie charts as is shown in the results section.

\section{Data Analysis}

The road network and crash data were loaded in the ArcGIS 10.1 software program witheach of the data points representing a single accident location. However there were cases where multiple crashes were reported to have occurred at the same location such as an intersection or near bus stops. Therefore, the crash data was aggregated to produce counts for each type of crashes at each location. GIS tools like proximity using Thiessens polygons and hot spot analysis tools were applied.

\section{GIS Proximity Analysis}

Defining the coverage or health facility catchment is vital in assessing population movements to health facilities. Accurate definition of these catchment areas is therefore important when defining targeted intervention measures. Different methods exists that can be used to define health facility catchment areas. First and the most accurate is to collect information at each health facility on where patients are coming from termed the patient flow method. The defined regions where patients come from to visits a certain health facility can then be approximated to be the catchment area of that facility (Zinszer et al. 2014). Its main disadvantage is however that the resources and time required to compile such a dataset for all health facilities makes it unfeasible. The second method is empirical, that involves collecting data on treatment seeking rates at different locations, and using the assumption that the nearest facilities are the ones used, catchment areas can be defined as is well documented in (Alegana et al. 2012).Third, there is the use of straight line distances such as Euclidean distances in GIS to define catchment areas. The disadvantage in this is that it leads to overlaps. These three methods are however only suitable in rural areas, and less is documented on their usability in urban areas. For urban areas we propose that Thiessen polygons are the most useful. This is because the tool splits the areas into regions assigned to each health facilities. Because the connectivity through roads in Nairobi is excellent, we did not expect much impact of land covers on accessibility. Finally, Voronoi diagrams are easy to generate and can be very useful tools for defining accessibility (Noor et al. 2006). This tool was used to give useful information of service areas that each of the health facilities served. This means it is possible to pinpoint the nearest health facility from an accident scene at a given location. All the health facilities in Nairobi and crash data locations were used in this proximity analysis. It should be noted that the nearest hospital to an accident scene might not be the best equipped to handle such cases.

\section{Hotspot Analysis}

This involved use of hotspot analysis tool in ARCGIS software. This tool was used to determine the accident hotspots for pedestrians in the study area.Before this tool was employed on the data, a severity index at 
each location was computed sinceit is difficult to know whether high or low clustering exists in 'unweighted' data. Counts of crashes are commonly used to evaluate safety problems at a location. Some experts believe that the more severe crashes should have greater weights in identifying unsafe locations on the basis of crash costs. Additionally, results of the crash severity method are sensitive to various weighting systems. According to (Geurts et al. 2004) there is no consensus on how the optimum weighting system should be developed; a compromise approach is becoming increasingly popular. The approach is to give weights to the more severe crashes, but not with the extreme high values computed in direct proportion to the crash costs. In this case, it was assumed that accidents involving pedestrians, cyclists, motorcyclists, passengers and vehicles higher weights in the decreasing order and be compounded by the fatality weight to gauge the overall severity of the accidents. Therefore fatal accidents involving pedestrians assumed the highest weight compared to the accidents involving vehicles and no fatalities involved. The weighted severity indices attribute was used in the derivation of the hotspot analysis map to show where more pedestrians related accident cases occurred.

\section{Spatial database creation}

The accidents database is a spatial referenced database with all data modeled as spatial objects and encoded in geospatial format, which stores all accidents related data in a central location, and provides functionalities to perform spatial query and spatial analysis and integration with other GIS platforms. It allows for query searches on various accident attributes in real time compared to the traditional accident database which is a summary spreadsheet format using general well known locations to denote location, type and severity of accidents.

This was created using Postgres-postgis database management system to assist in future data editing and implementation of the accidents management system. This provides an easier and free platform to manage spatial data perform queries, update data and give real time reports. It was also meant to be interfaced with accident management and monitoring web application that happened to be a side product of this research.From the databases, it is possible to visualize accident historical trends and to identify and pin point accident-prone locations.An example of a query run to show the nearest health facilities from a selected accident spot at Kangemi yielded the following as shown in the Figure 1 below:-

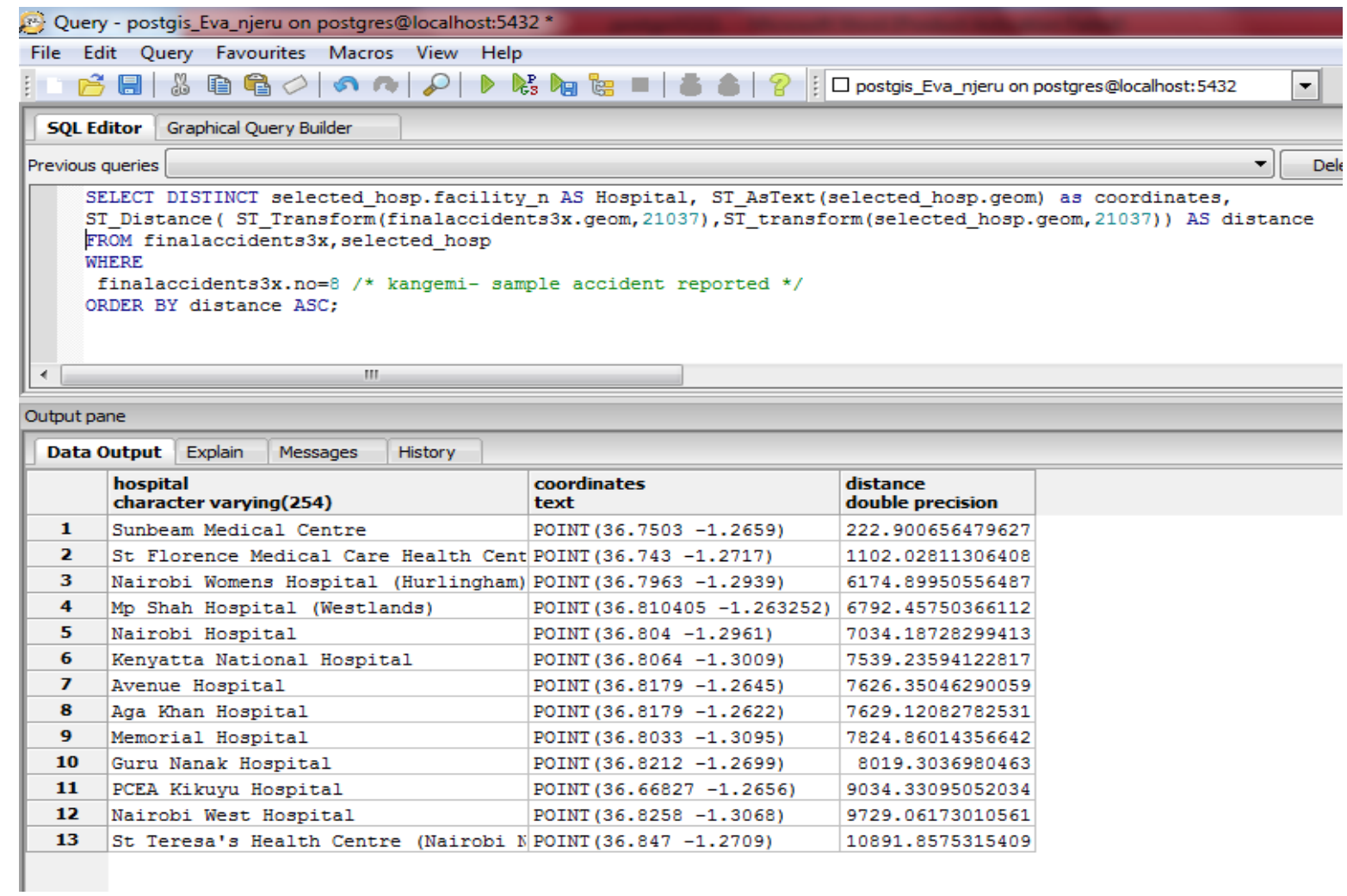

Figure 1: Using Postgres-postgis to find the nearest health facility

\section{Production of Maps and Reports}

After the analysis were done, hotspot and proximity maps were produced. To enhance better interpretations of the products, charts were also created to assist in map reading and interpretation. 


\section{Development of Accident Information Management System (AIMS)}

The Accident database was developed and interfaced with prototype accident information management system (AIMS). The components of the system are;

- Spatial databases: To manage and query data

- Web forms: For adding new data and data filtering

- Mapping APIs: Leaflet, mapbox (uses javascript to load maps onto the web page)

- Server side script: To process request and link postgres to web application

- Geojson data format (altenative to KML)

\section{Results}

It was found that the collected information about the accidents are entered by the attending officer and stored in the hard-covered books at the police stations. There were no police officers who had specifically been trained to handle accident data in terms of data entry, processing, analysis and interpretation.Probably the compromised quality of the data may be attributed to the quality of information collected on particular accident events and failure to recognize that such information is important for road safety planning and policy interventions. This results to incomplete and sometimes inaccurate accident data. Another problem of poor data quality could be due to accidents underreporting and lack of regular updating of the information in the records.

\section{Accidents by Location}

A comprehensive database of accidents occurring on Waiyaki way in Nairobi was compiled. There was a significant variation in accident occurrence along the road with hotspots clearly evident at Kangemi area and Cooperation stage. Figure 2 below summarizes these results.

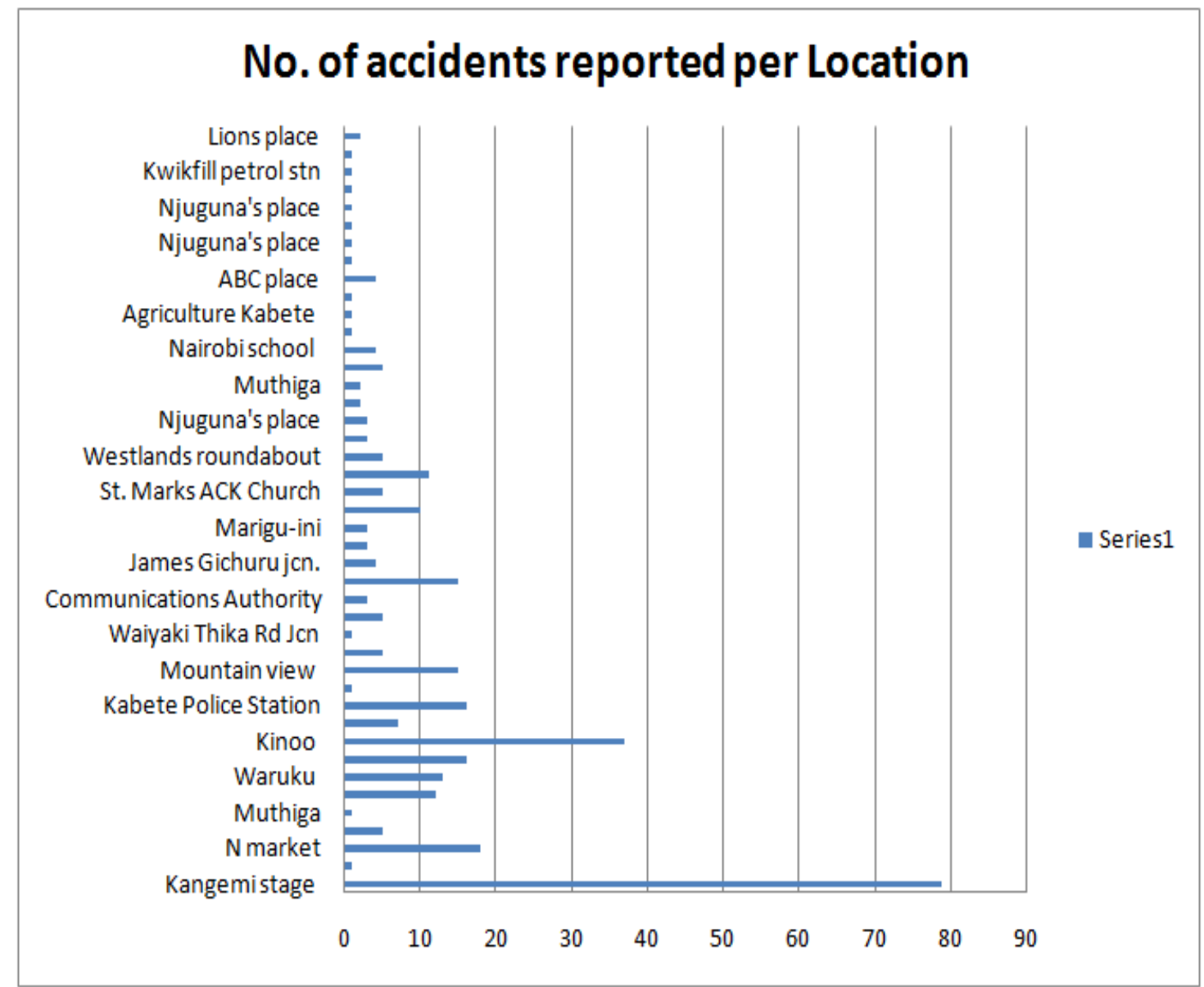

Figure 2: Accident incidents per location, the chart that portrays Kangemi as an accident hotspot having reported many cases of accidents than any other location.

\section{Nature of the Accidents}

Figure 3 below is a pie chart showing the relative numbers of various accident victims/actors in the reported accidents. From this, it is clear that the pedestrians form the biggest group of victims in any reported accidents. 


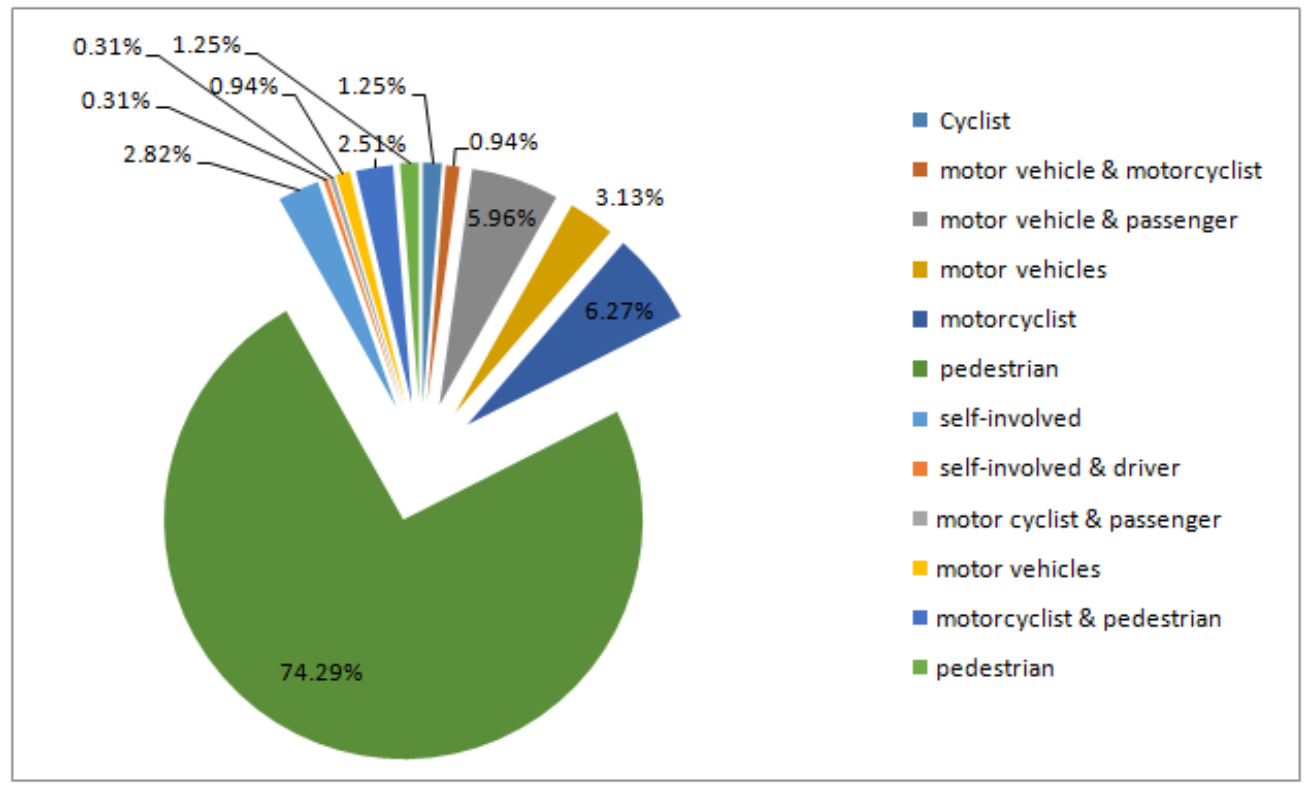

Figure 3: Number of Victims involved in the accidents

\section{Analysis of Temporal Patterns of Accidents}

Figure 4below displays the relationship between accident occurrences and time of the day. The figure depicts that most accidents that were reported happened during the evening hours (4pm - 10pm) with significant occurrences in both early morning and late night.

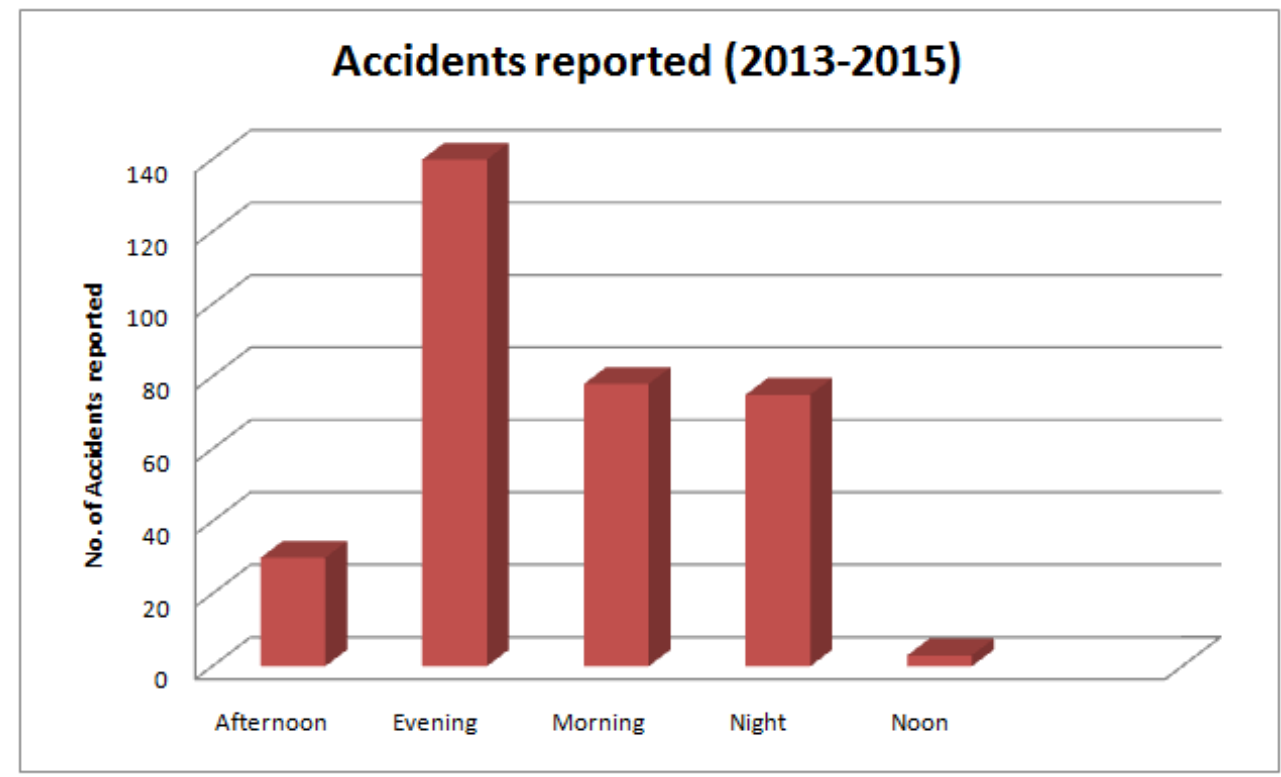

Figure 4: Bar chart showing prevalence of accidents vs time of the day

\section{Hotspot Analysis}

Step 1: Moran's I Index tool in ArcGIS was used to measure spatial autocorrelation based on both locations and values of features to investigate the spatial patterns of the accidents. At a distance threshold of 800 meters, the Z-Score reached the highest value of 0.607881 which means that the accident data is clustered until the distance of a threshold of 800 meters with a statistical significance level of 0.01 .

Step 2: Getis-Ord statistic (ArcGIS tool) was used to identify accident hotspots. A high value of GetisOrd statistic represents a cluster of high index values (hotspots) while a low value represents a cluster of low index values (coldspots). The distance threshold of 800 meters that was associated with maximum Z-Sore in step 1 was then chosen for the Getis-Ord analysis using the hotspot analysis. These results are summarized in Figure 5 below. 


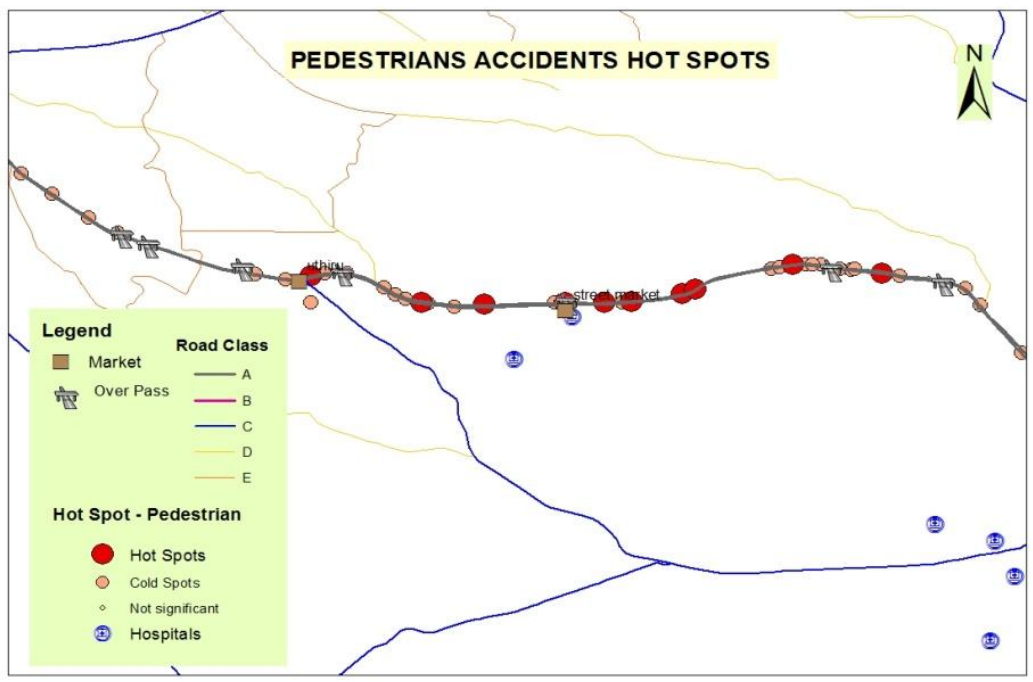

Figure 5: A map showing pedestrian accident hotspots

\section{Health Facilities to Accidents Response}

Figure 6 shows the proximity to health centres. It was noted that the chosen facility did not coincide with the nearest facility. This was attributed to differences in facility staffing and equipment and costs to the victims.

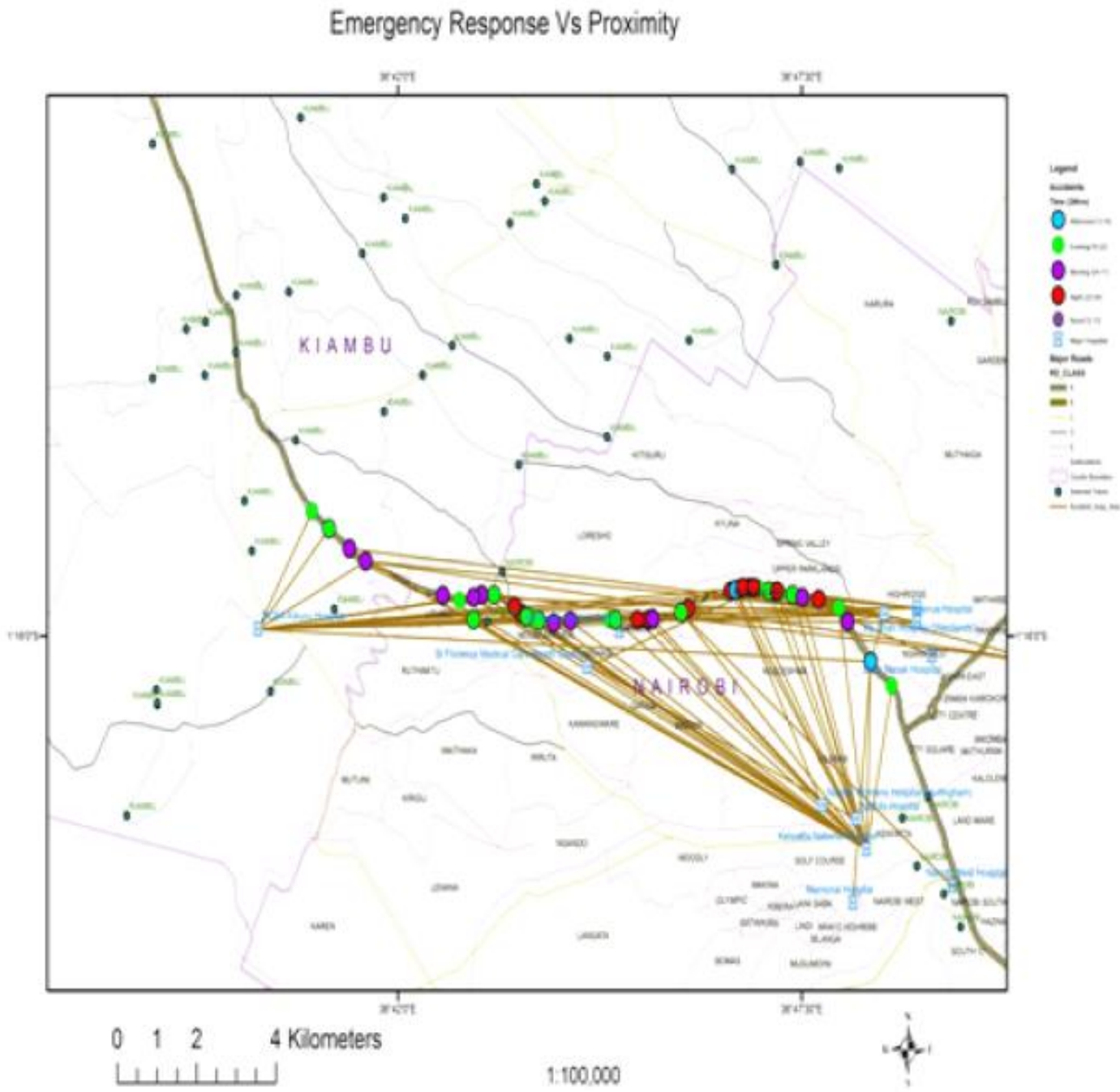

Figure 6: A map showing proximity to health facilities 
Health facilities within the study area were further ranked in relation to their capabilities in accident responsiveness. Dispensaries and clinics were catogorised as ideal for minor injuries since they nearer the accident hotspot areas and they are also not adequately equipped to handle serious injuries. Referral hospitals were classified as ideal for serious injuries since they are well equipped with personnel and facilities. The ranking is summarized here below:-

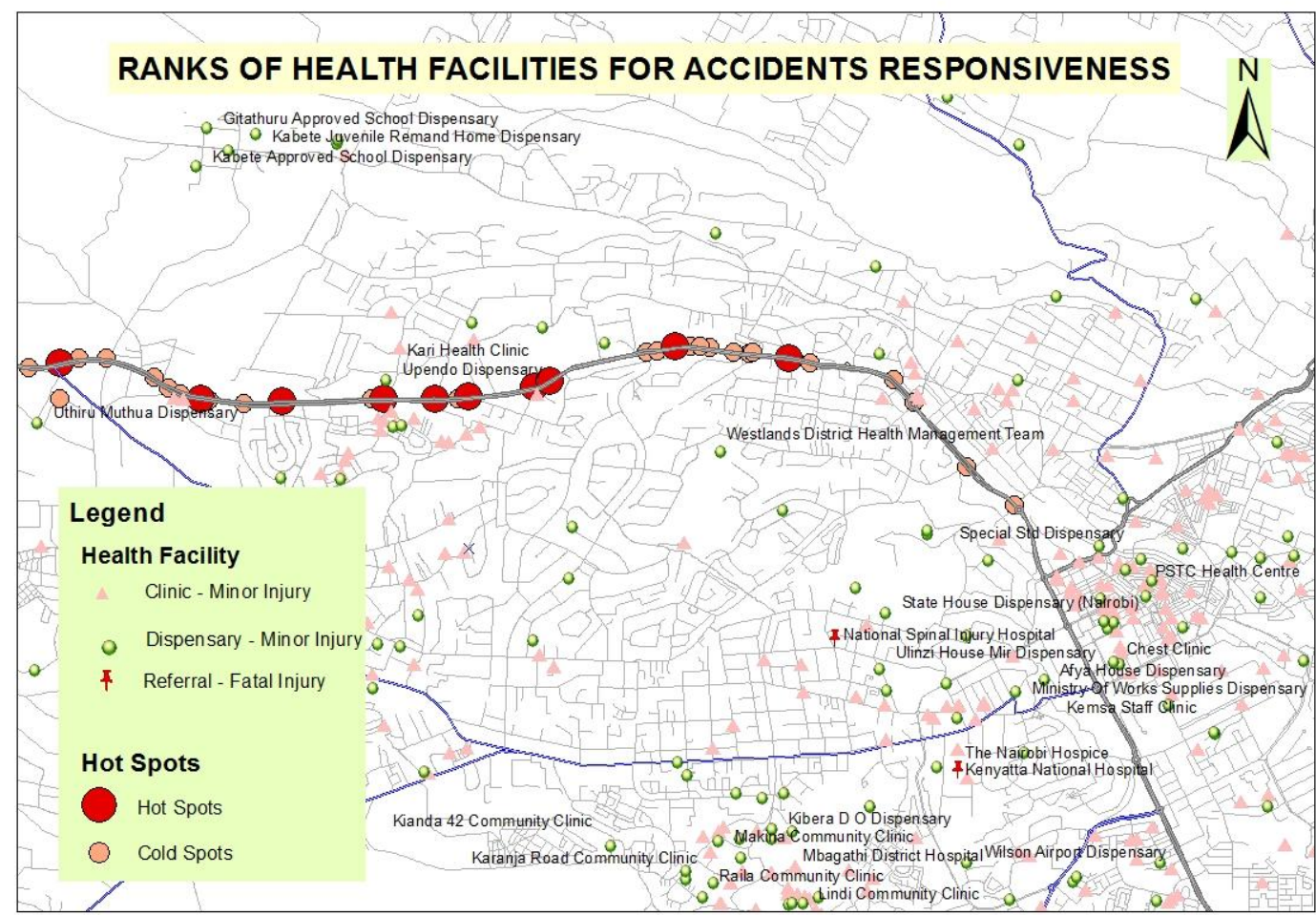

Figure 7: Ranking of health facilities for accident responsiveness

\section{Bus stops to Accidents Comparison}

This study adopted a stopping distance of $100 \mathrm{~m}$ from the bus stops to determine the ranks of the bus stops in relation to the accident events. The results indicated that the bus stops at Kawangware junction and Kangemi had the highest number of accidents followed by those at Sodom and Uthiru areas. Kinoo, 87, Kabete Barracks and Westlands bus stops recorded low number of accident events. These results are summarized in Figure 8 below:-

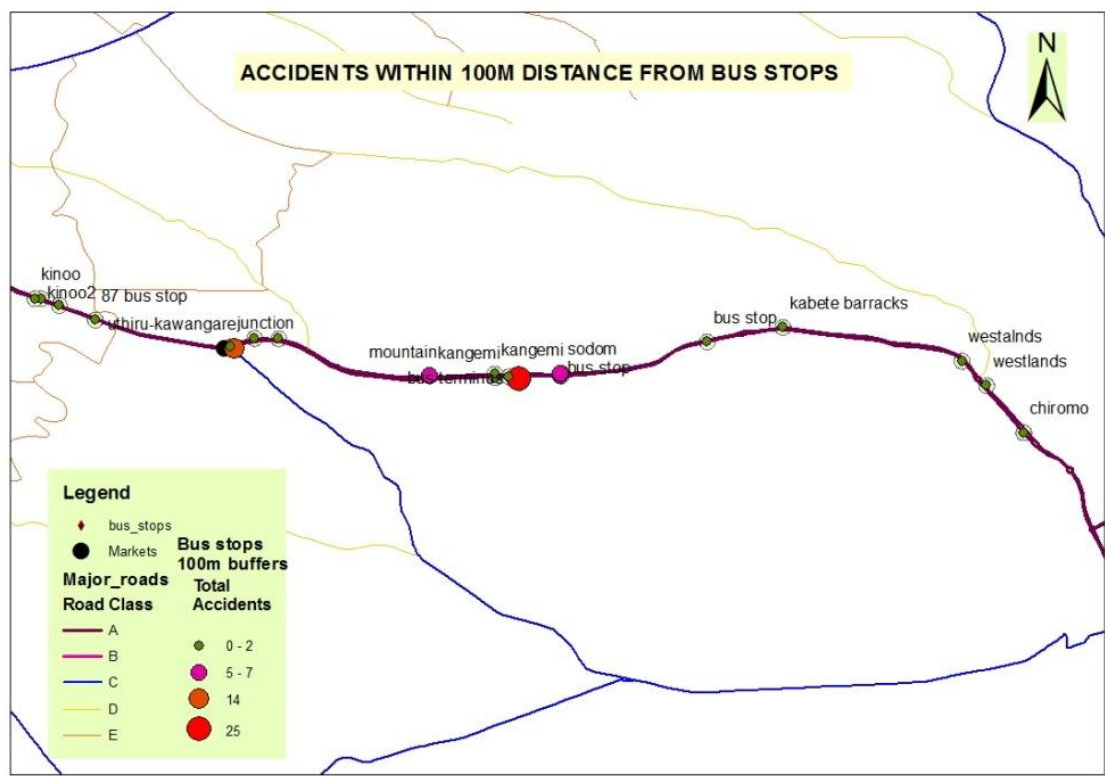

Figure 8: Bus stops to accidents correlation 


\section{Correlation of Road Accident Locations to School Proximity}

The research adopted a distance of $500 \mathrm{~m}$ as the walking distance to a facility or service provider. A buffer zone of $500 \mathrm{~m}$ was therefore used to determine the schools near Waiyaki Way. The results on Figure 9 below shows that the areas with high number of accidents also displayed a high number of schools within this buffer zone.

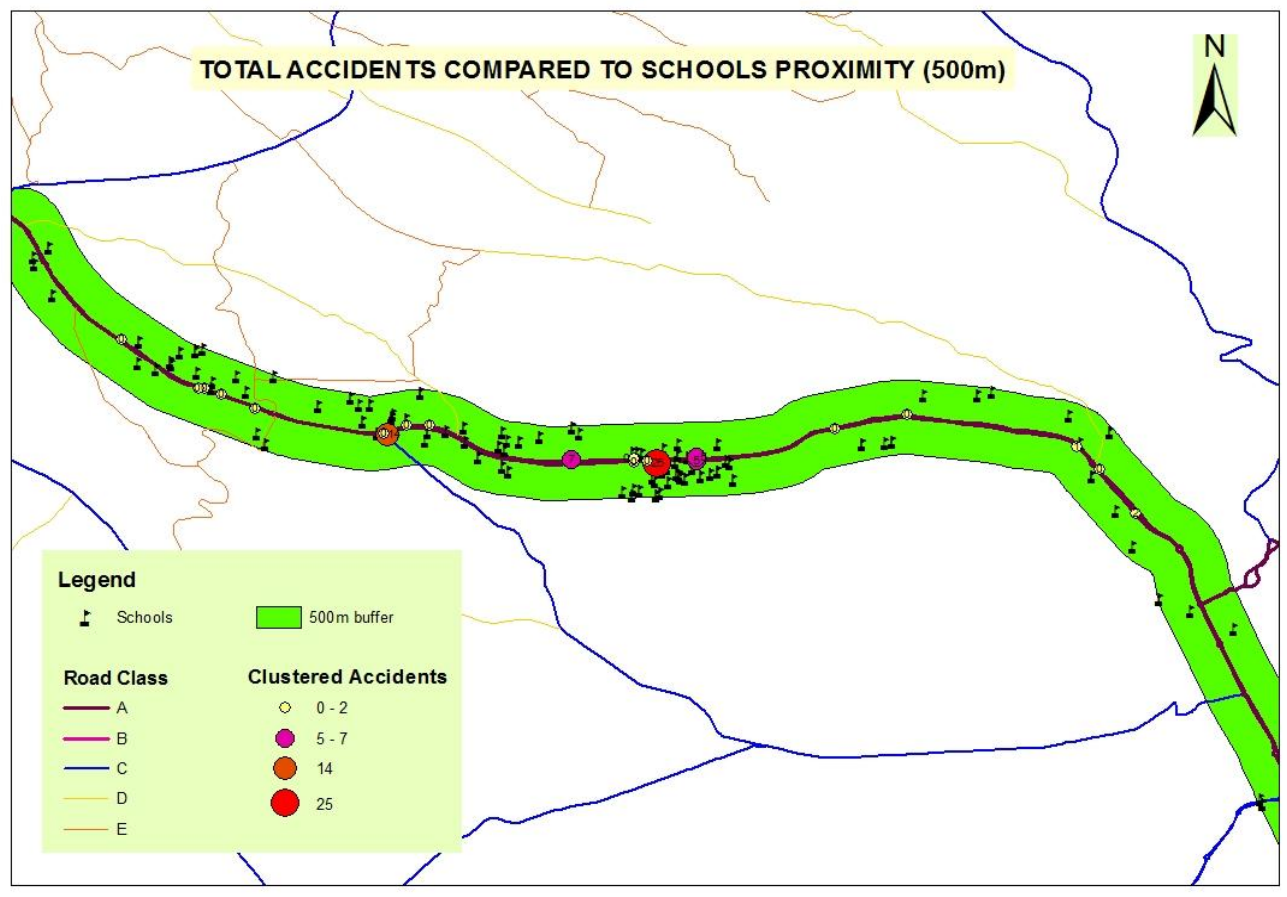

Figure 9: Schools correlation to accident locations

\section{Dot Density of Accidents to Overpasses and Street Markets}

The dot density analysis was applied to correlate the accidents to overpasses. The results as summarised in Figure 10showed that the overpasses at Kawangware junction and Kangemi areas had high dot density rating them as unsafe overpasses. The two overpasses also have street markets nearby thus contributing to high population around these areas.

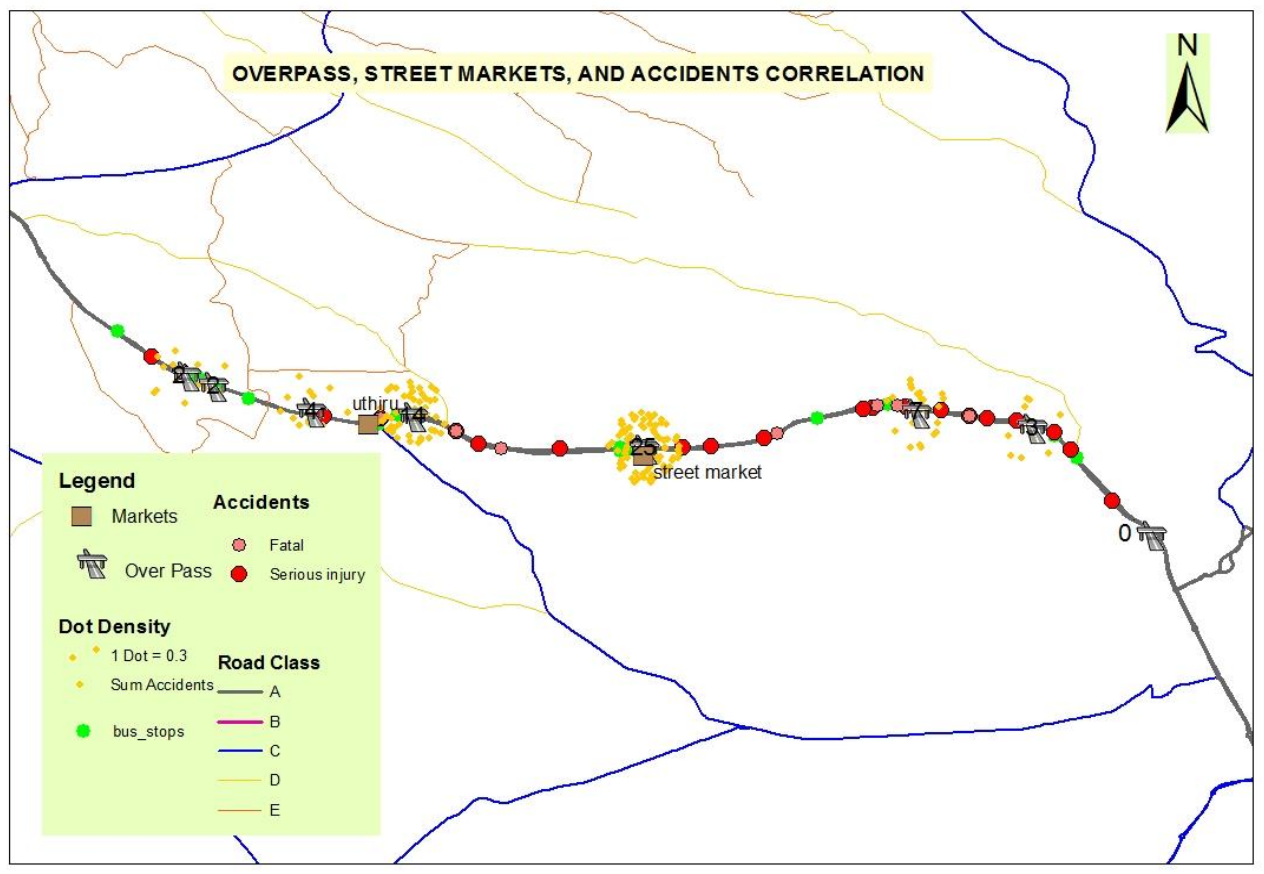

Figure 10: Correlation of accidents with overpasses and street markets 


\section{Development of Accident Information Management System (AIMS)}

An attempt was made at developing a GIS-database-driven web application for accident reporting and management. This system could assist in real time accident recording, retrieval updates, visualization, production of reports and also management of the victims through identification of the most suitable facility and route for emergency medical services. The system proposes that the public could report geo-referenced accident incidences to the system as well as the police. The data acquired and kept in the database could be exported to common GIS softwares for further processing, trend analysis, data sharing and visualization.

The system architecture shown in Figure 11 represents both the front and back end of the system. The spatial database handled the spatial data and queries. The databases contained datasets on accidents, health facilities and road network.

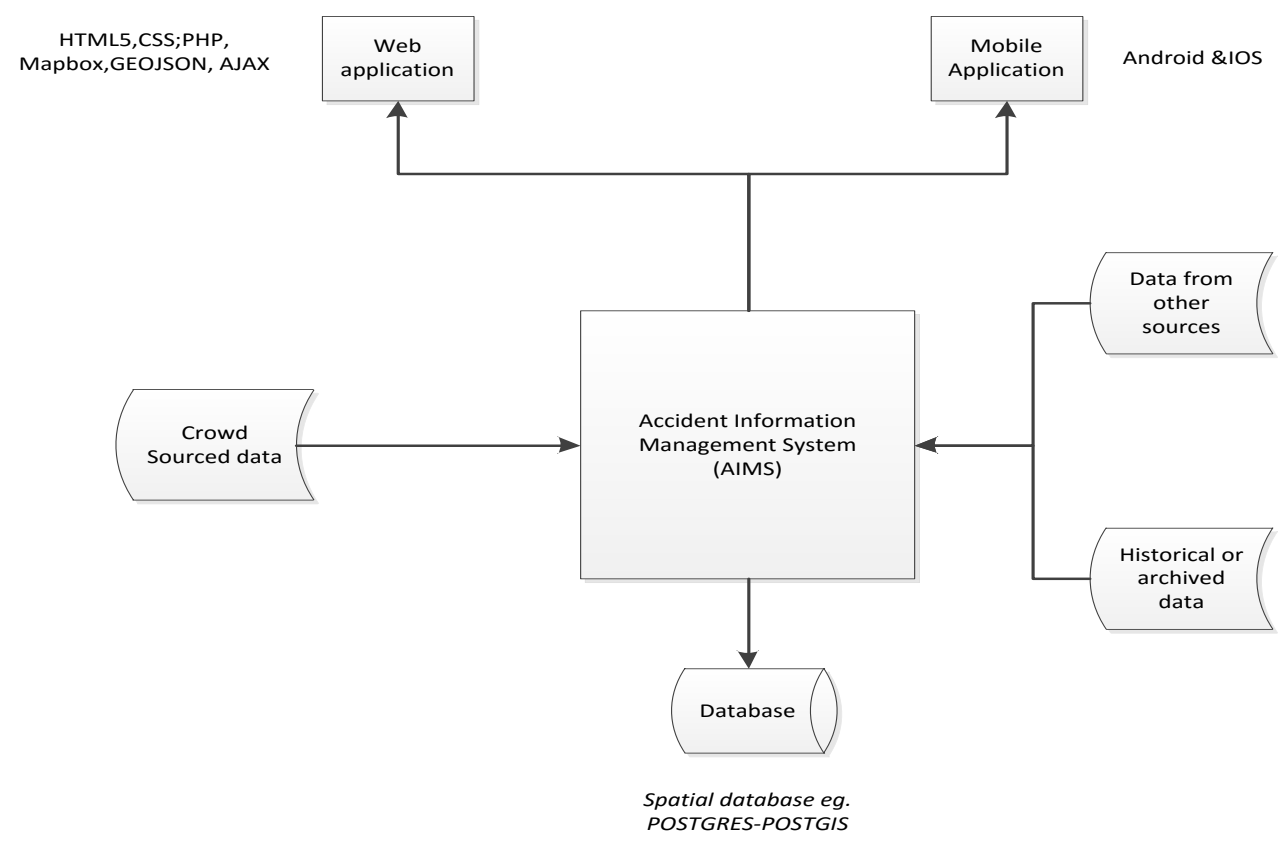

Figure 11: System Architecture

Figures 12, 13, 14 and 15 demonstrates the user-interface part of the system through which the users can be able to interact with the web application. The interface provides for commands to the program and display of information on the screen.

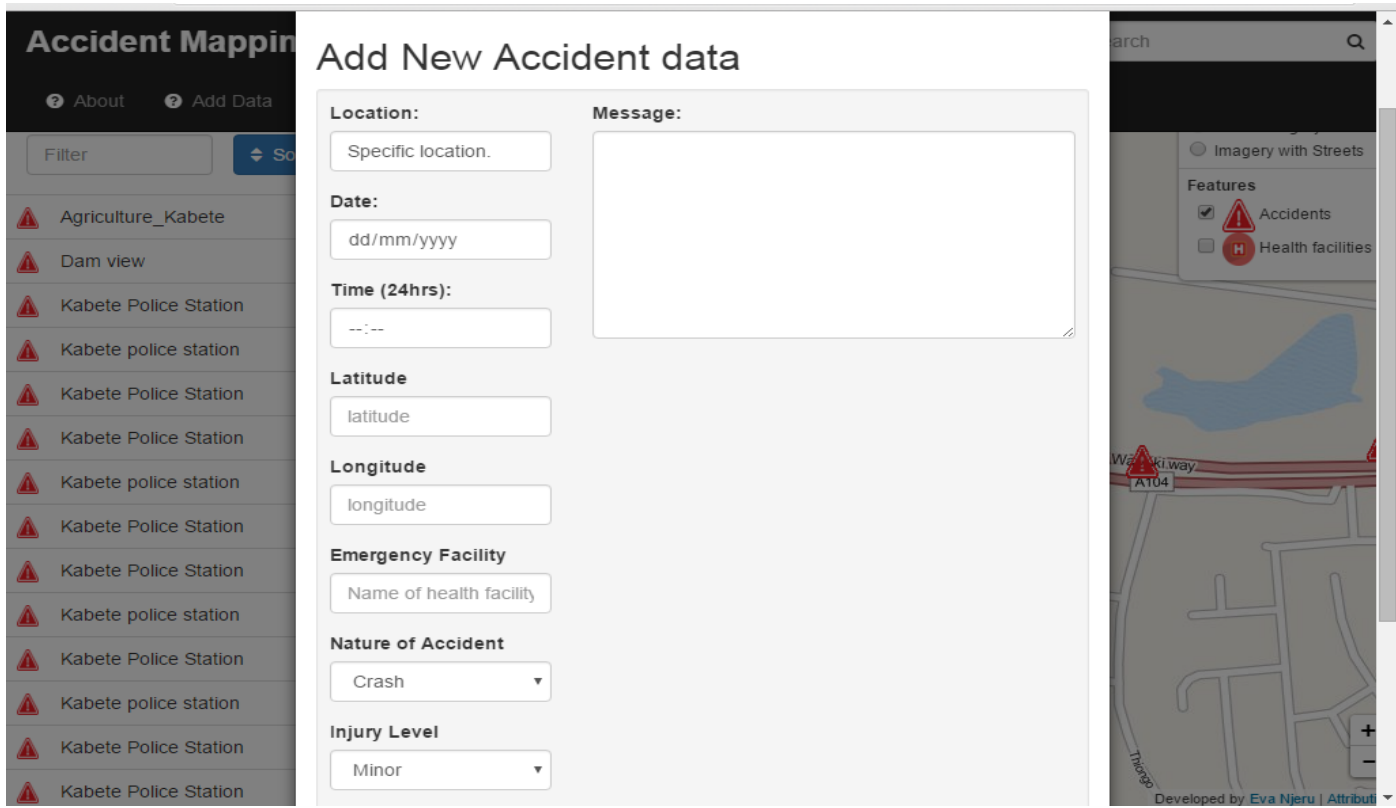

Figure 12:A screen shot of AIMS prototype showing the user menu 


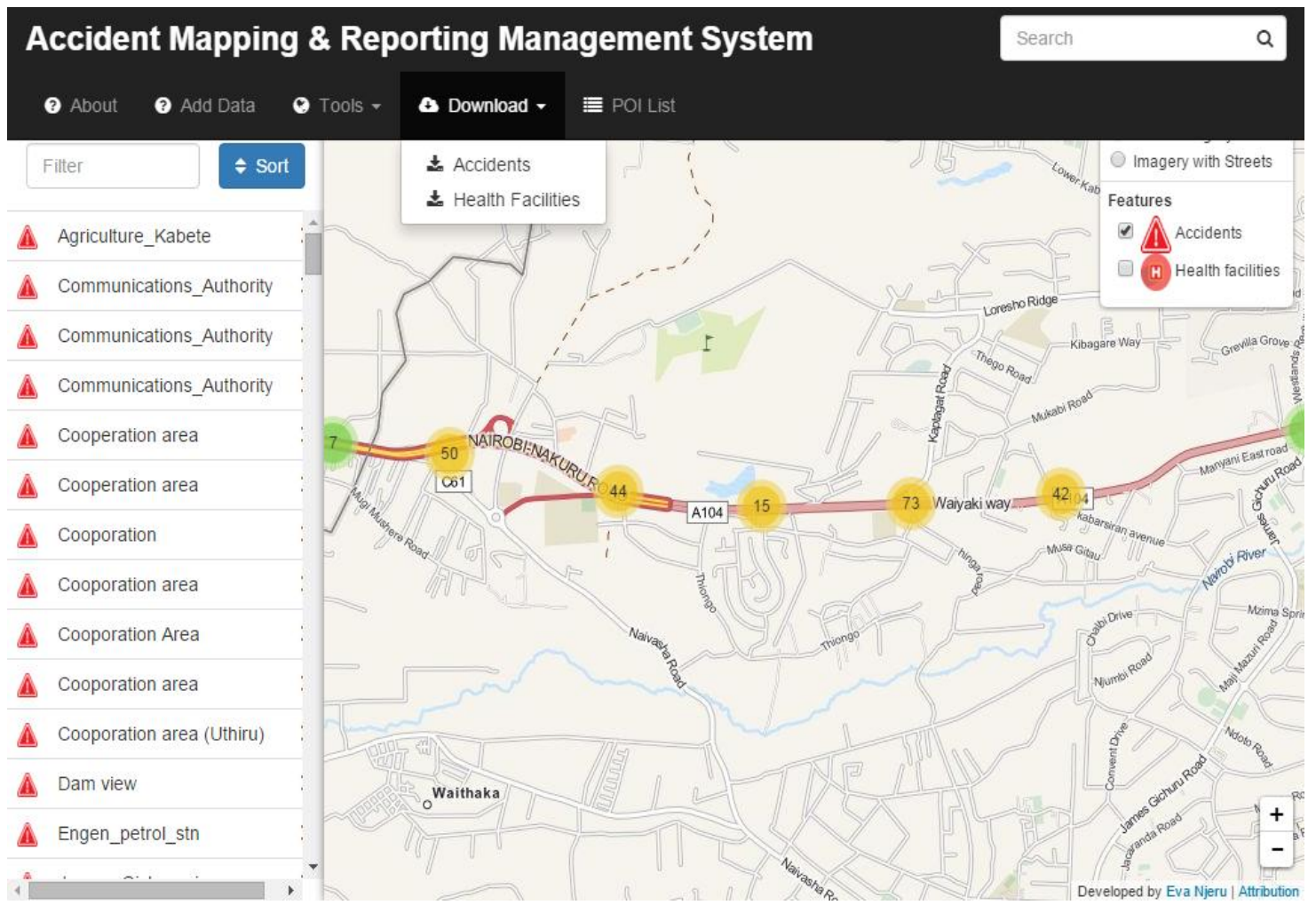

Figure 13: A screen shot of AIMS prototype showing user menu and graphical user interface

\section{Accident Mapping \& Reporting Management System \\ Q}

\section{(3) About 3 Add Data 0 Tools - 4 Download - E POI List}

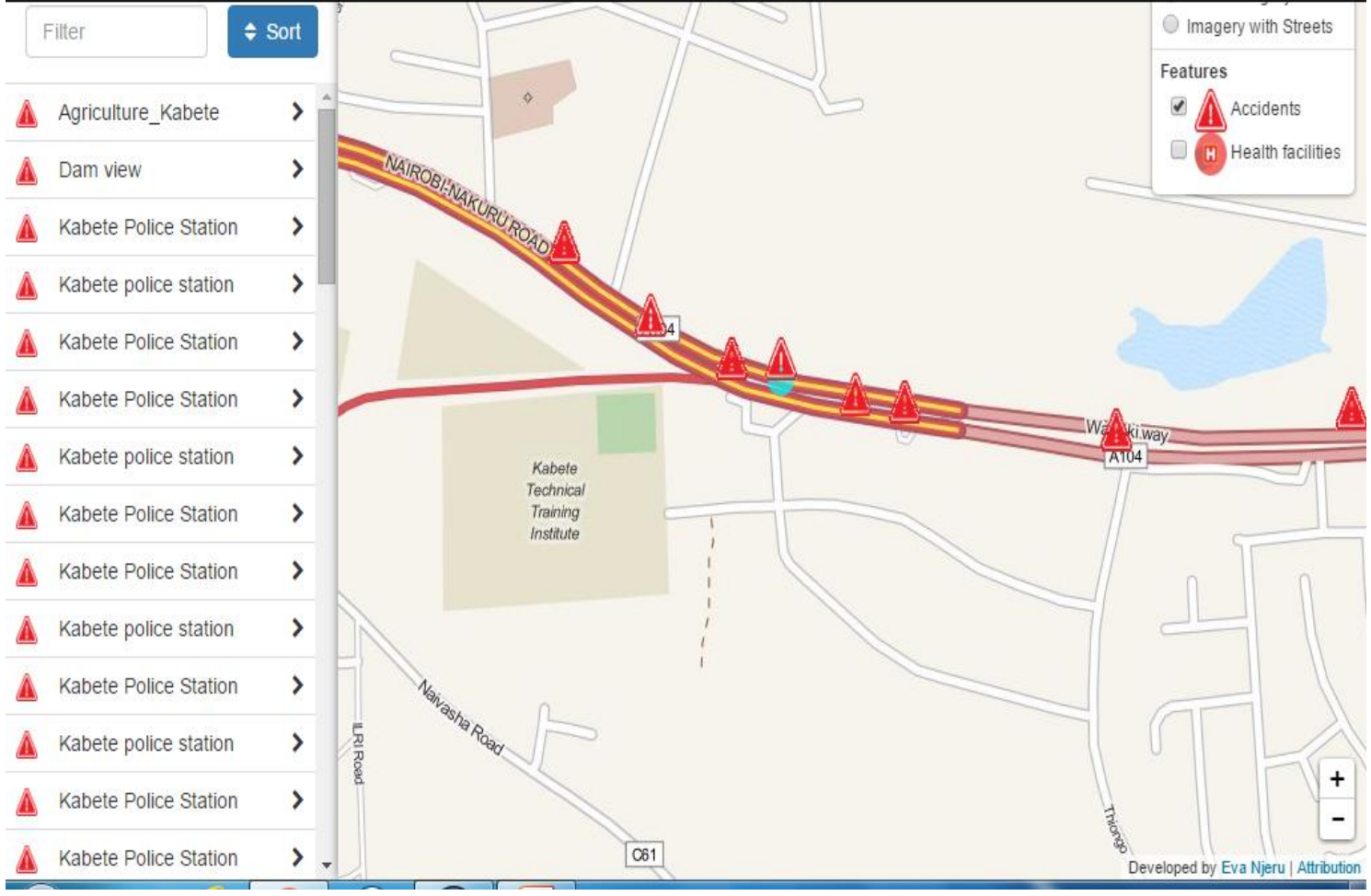

Figure 14: A screen shot of AIMS prototype showing user menu and graphical user interface

Figure 15 below is a screen shot of the AIMS showing how the system can help in establishing the route to the nearest health facilities from locations of the accident events. 


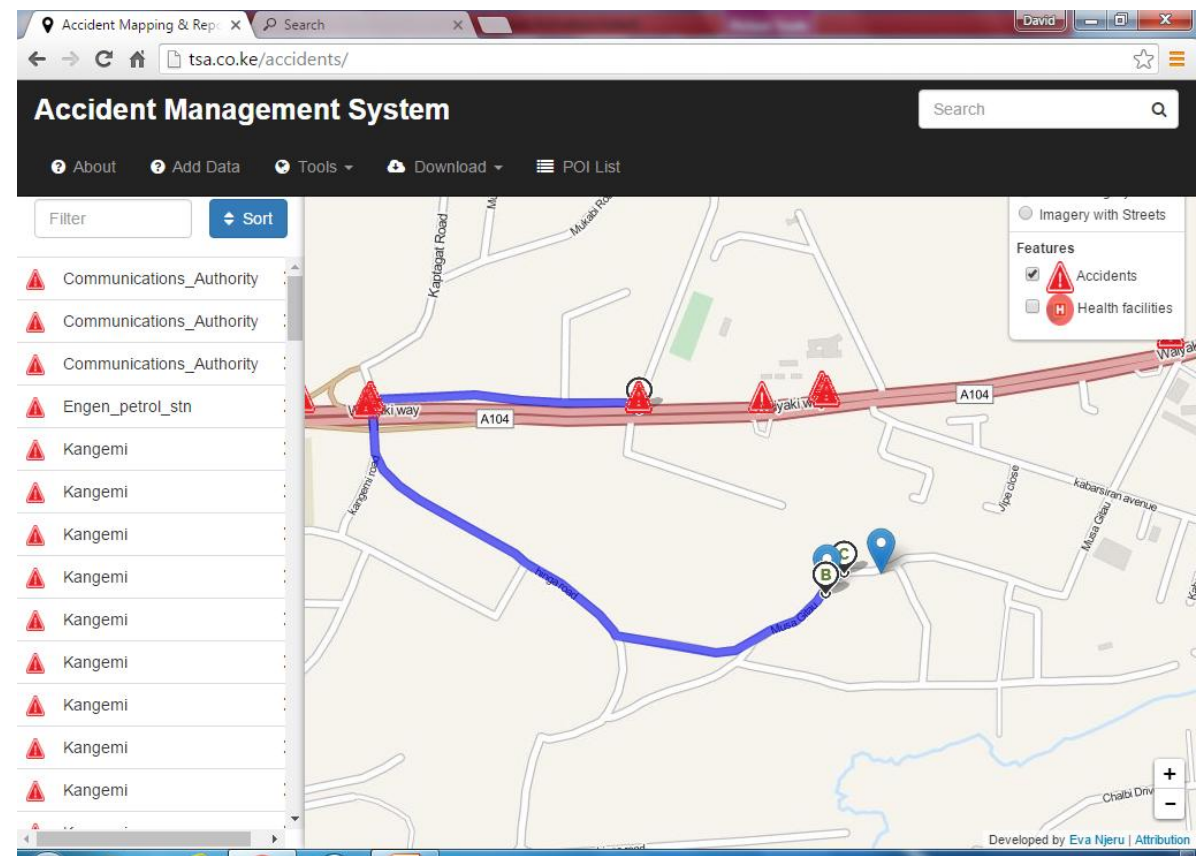

Figure 15: A screen shot of AIMS prototype showing routes to nearest emergency services

\section{Discussion}

This project intended to develop and present a system that can be used for accident management, through collection and analysis of accident data including the viability of emergency services. A database of accident incidences and their locations was successfully assembled. Accident hotspots were defined in a GIS system and analysis of hotspots was highlighted. Together with the location of the health facilities, the catchment areas and access to health facilities was also defined for the area of study. Most importantly, a web system was generated that can be accessible from any location. The system therefore shows the location of accidents over the course of the last three years, and various analytic results such as the nearest health facilities. The implications of such study are manifold. First the visualization capabilities of the outputs is quite useful when accident hotspots are to be seen easily for timely responses. Currently, the police in Kenya uses a system where accident data are recorded on papers which are not only tedious but also not easy to analyze. By converting such datasets to computer readable formats, thenmuch more important information can be derived to make meaningful decisions. Clearly, we show thatthe greatest burden of accidents occurs at the Kangemi stage, which accounts for a high percentage of all the accident locations. In addition, a great number of persons involved in the accidents are the pedestrians, accounting for more than $75 \%$. From the two pieces of information we postulate that at Kangemi stage, there could be many pedestrians crossing at illegal locations or standing in areas which make them vulnerable to the accidents. Therefore the decision makers should consider putting up a footbridge or a better bus terminus to prevent such fatalities. Finally, for mitigation purposes, areas with similar characteristics then call for similar measures to prevent future obnoxious implications.

Secondly, the study shows that spatial analytic tools can be useful for assessing and displaying accident data. The maps produced can easily be visualized by traffic authorities when making decisions. The hotspot analysis is definitely an important tool as it was able to show areas that are 'hot' due to accidents in addition to the 'cold' areas. As we have proposed earlier, then interventions can be easily targeted once the hotspots are easily identified. Relating accident events to public utilities and service areas like schools, markets, bus stops and overpasses gives insight into the contribution of such services to accident occurrences. This can help in determining the most suitable locations for the service facilities in relation to roads. In addition, relationships of such hotspots to proximity to health facilities provides an important result. Here we saw that the health facilities which are nearest can be identified to help in management of future accident victims. We recommend that traffic decision makers should utilize this important tool to avoid serious fatalities of accidents. Such outputs can extend to ambulatory services, for example, having them on standby at specific locations so that they can easily respond to cases needing responsive care. Finally, we hope to disseminate these results to traffic commandant in charge of the study area to find utility to the outputs.Thirdly and most importantly, we propose the development of web based-system that can be used for data dissemination across various sectors. For example, in Nairobi, various stakeholders include the traffic police, the Kenyared cross, the St Johns Society and the hospitals with ambulatory services. These actors can only be interlinked with a system that is able to accommodate variation in space such as a web based arrangement. This can be helpful especially when coordinated response is required 
from different departments. As such, our web based system provides a novel approach that can be used for handling accident data by various sectors. Our study was not devoid of limitations. First, our accident data source is only from those recorded by the police. We understand that there could be more information on accidents not recorded by the police as response was arrived at much earlier. We hope that extending the AIMS to a multidisciplinary system can help in data collection from various sectors. Secondly our accessibility analysis did not include travel impedances such as traffic jams within the city. We believe that including such as aspect of travelling within the city would improve the results. Finally, a time series analysis of the accident data would have also improved the information we are able to display. Despite the availability of data on the time of the accidents, we recognize that such an analysis would require inclusion of much more information into a model, an area that would form an interesting area of study in the future. The study can be extended to other roads in the future, and similar analysis carried out. Future studies should therefore focus on analyzing such information at a wider spatial scale, especially for interlinked roads within the city. In addition, a time series model that uses much more information such as socio-economic status of the people, time of accidents, proximity to alcohol selling points among others would form a very interesting aspect of study. Geospatial techniques of GIS supplemented with GPS are recommended to improve the current system on data collection, storage, manipulation and analysis of accident data to aid in decision making. This study recommends use of accurate geo locations for accident scenes to avoid multiplicity of accidents on one generalized location for better analysis. This could be done by equipping police with GPS devices or at least location enabled mobile phones with a dedicated application to capture accidents. It also recommends the adoption of real-time accident reporting system to leveraging technological advances in web and Mobile GIS.Modern smart phones come with inbuilt GPS hardware. This could be used in capturing and geo-tagging accident scenes and relaying this data to central AIMS server and to emergency responders in real time. This will improve the data quality and data storage constraints experienced today at the police department. This data can then be shared with experts, decision makers to respond to the accident cases. This data can also be used for further analytical processing and visualizations for better future accident related researches

\section{Conclusion}

The current accident reporting mechanisms and system can be revamped to capture accurate geographical locations as one of the key attributes. This can then be done through GIS where it would involve mapping the accident areas and visually analyzing the data. This information can be combined using GIS applications and analyzed for clusters, proximity and trends. Today, the use of Geographical Information System (GIS) for accident data analysis is not widely used in the police department. This is because the data is analyzed on a computer without locating the incident spatially. The ability to analyze the data visually using spatial GIS is most likely to give the analysts a clear understanding of the accident. By use of GIS, the time and effort required to analyse accident data can be reduced.This study could be extended to cover wider area for more accurate reliable results.

\section{References}

[1]. Alegana VA, Wright JA, Nahzat SM, Butt W, Sediqi AW, Habib N, et al. Modelling the incidence of Plasmodium vivax and Plasmodium falciparum malaria in Afghanistan 2006-2009. PLoS One. 2014;9(7).

[2]. Alegana VA, Wright JA, Pentrina U, Noor AM, Snow RW, Atkinson PM. Spatial modelling of healthcare utilisation for treatment of fever in Namibia. Int. J. Health Geogr. [Internet]. 2012;11(1):6. Available from: http://www.ij-health geo graphics.com/content/11/1/6

[3]. Geurts K, Wets G, Brijs T, Karlis D, Vanhoof K. Ranking and Selecting Dangerous Accident Locations : Correcting for the Number of Passengers and Bayesian Ranking Plots. 2004;

[4]. Lagarde E. Road Traffi c Injury Is an Escalating Burden. 2007;4(6):967-71.

[5]. Manyara CG. COMBATING ROAD TRAFFIC ACCIDENTS IN KENYA: A CHALLENGE FOR AN EMERGING ECONOMY. $2015 ; 1-7$.

[6]. Muchene LK. Road accidents in Kenya : a case of poor road network or human error ? 2014;

[7]. Nagne AD, Gawali BW. T RANSPORTATION N ETWORK A NALYSIS B Y U SING R EMOTE. 2013;3(3):70-6.

[8]. Noor AM, Alegana VA, Gething PW, Snow RW. A spatial national health facility database for public health sector planning in Kenya in 2008. Int. J. Health Geogr. [Internet]. 2009 Jan [cited 2015 Mar 9];8(1):13. Available from: http://www.ijhealthgeographics.com/content/8/1/13

[9]. Noor AM, Amin A a., Gething PW, Atkinson PM, Hay SI, Snow RW. Modelling distances travelled to government health services in Kenya. Trop. Med. Int. Heal. 2006;11(2):188-96.

[10]. Odero W, Garner P, Zwi A. Road traffic injuries in developing countries: a comprehensive review of epidemiological studies. 1997;1997(5):445-60.

[11]. Ouma PO, Odera PA, Mukundi JB. Spatial Modelling of Weather Variables for Plant Disease Applications in Mwea Region. J. Geosci. Environ. Prot. 2016;(May):127-36.

[12]. Rudtanasudjatum K. Road Traffic Accidents Management Model : The Successful. 2008;1-4.

[13]. WHO. Global status report on road safety. 2009;

[14]. Zinszer K, Charland K, Kigozi R, Dorsey G, Kamya MR, Buckeridge DL. Determining health-care facility catchment areas in Uganda using data on malaria-related visits. Bull. World Health Organ. [Internet]. 2014;92(October 2013):178-86. Available from: http://www.pubmedcentral.nih.gov/articlerender.fcgi?artid=3949593\&tool=pmcentrez\&rendertype=abstract 